\title{
Role of non-collinear magnetization: from ferromagnetic nano wires to quantum rings
}

\author{
Nicholas Sedlmayr ${ }^{*}, 1$, Vitalii Dugaev ${ }^{2,3}$, Jamal Berakdar $^{1}$ \\ ${ }^{1}$ Department of Physics, Martin-Luther-Universität Halle-Wittenberg, Heinrich-Damerow-Str. 4, 06120, Halle, Germany \\ ${ }^{2}$ Department of Physics, Rzeszów University of Technology, al. Powstańców Warszawy 6, 35-959 Rzeszów, Poland \\ ${ }^{3}$ Department of Physics and CFIF, Instituto Superior Técnico, TU Lisbon, av. Rovisco Pais, 1049-001, Lisbon, Portugal
}

Received XXXX, revised XXXX, accepted XXXX

Published online XXXX

PACS 75.60.Ch,75.75.+a

* Corresponding author: e-mail sedlmayr@physik.uni-kl.de

We consider interaction effects related to a nonuniform magnetization in ferromagnetic nanowires and their possible generalization to nanorings. First we show that the electric current in a ferromagnetic nanowire with more than one domain wall induces an exchange coupling between the walls mediated by the spin-dependent interference of scattered carriers. This interaction reveals a complex behavior as a function of mutual orientations and separation of the domain walls, thus affecting the domain wall dynamics. Then we consider how the theory should be modified in the case of a magnetized quantum ring. In this case the situation is more complicated because the vortex and onion magnetization states in the ring are not truly ferromagnetic.

Copyright line will be provided by the publisher

1 Introduction Domain walls (DWs) are noncollinear magnetization regions separating areas of different homogenous magnetizations. In nanostructures they are the subject of numerous investigations and are also important for applications [1, 2,3]. In low dimensional nanostructures, such as the wires and rings we are here concerned with, the coupling between the electrons and the DWs is enhanced in comparison with the bulk scenario [4]. This can drastically alter the transport properties of the nanostructures [5, 6,7]. The spin flip of the carriers as they traverse the region of non-collinearity leads to a spin-torque acting on the DW, and consequently to the possibility of current-induced DW motion [1,2].

DWs can exist not only in magnetic nanowires but can also be created in magnetic nanorings [8,9] between the regions of a locally defined homogenous magnetization. Fully ferromagnetic rings, where the magnetization direction is globally preserved, are not experimentally found, though they inform the majority of theoretical considerations. Instead, the magnetization direction usually follows the shape of the annulus. In this case it is clear that the DWs always come in pairs and as such a natural question to ask is how they interact, mediated by the electrons in the system. As a precursor to this question we discuss such interactions, and their effect on the DWs in nanowires [10 [1]. This will form the bulk of the results presented in this paper. The case of quantum dot with a non-collinear magnetic order has been discussed in [12]. Before addressing the case of magnetic rings, we discuss several fundamental points. In addition to the effects of electrons on the DWs we are also interested in how the ferromagnetic nature of the system and the presence of the DWs affect such wellknown phenomena as the Aharonov-Bohm effect [13,14], persistent currents [15, 16], and conductance [17].

It is established that strong carrier scattering and interference results in long-range interactions between impurities on metal surfaces. The question of whether and how the carriers' spin dependent scattering mediates interactions between DWs is addressed here. We have found that the DWs are coupled thusly: due to the scattering from

\footnotetext{
${ }^{1}$ This can be explained by simple magnetostatics accounting for the demagnetization fields.
} 
the first DW a spiral spin density wave is created. This then acts as a spatially non-uniform torque on the second DW whose energetically stable shape and position therefore has a non-uniform dependence on the distance from the first DW. This is different from the ordinary spin-torque transfer in bulk spin valve systems [18, 19,20] or magnetic tunnel junctions [21,22] insofar as in this case the DWs spatial arrangement, in addition to the magnetization direction, is current controlled.

There are a number of experimental works on permalloy ferromagnetic rings [8,9]. In these works the existence of the Aharonov-Bohm effect was experimentally confirmed [23,24] and the studies of the magnetoresistance effect have also been presented [25, 26, 27]. However, it is still not clear how the variation of magnetization in these systems affects the state and dynamics of the DWs. Though our aim is to consider DWs in the nanoscale rings, we discuss first the rings without DWs. The main question is how the vortex state in a magnetic ring affects its electric properties. In particular, how the corresponding edge states look like and how persistent currents and Aharonov-Bohm type effects are modified by choosing different magnetization configuration.

The first question to answer involves analyzing the electron wavefunctions of the ferromagnetic ring [28, 29]. Note that in the following we call the magnetization of ring "ferromagnetic" when the magnetization vector is directed along the $\theta$ angular unit vector in the cylindric coordinates of the ring. In this configuration there is no energy cost from the demagnetization field, unlike the true ferromagnetic case. The vortex state instead incurs the smaller penalty of not having exactly parallel magnetic moments everywhere, though locally they will be approximately parallel for a large ring. Note also that as we are dealing with a ring, there is no energy cost due to the vortex core.

The Zeeman splitting of the electron spectrum in a magnetic field has been included in Refs. [30, 31]. The overall effect of this, as far as the Aharonov-Bohm effect is concerned, is to alter the dephasing length scales. The spin-orbit coupling has also been included but only perturbatively where it mixes the spin channels. Though the spin-orbit coupling could be considered analogous to what we wish to calculate (which also couples angular orbital momentum and spin) we solve this model exactly.

Another interesting idea concerns the magnetically textured mesoscopic ring of Ref. [32]. In this model, one considers the inhomogeneous magnetic field distorted from the $z$-direction around the ring, like a crown. This induces a form of spin-orbit coupling which can lead to persistent currents even in the absence of a magnetic flux threading the ring. The method can be useful for decoupling the orbital and spin degrees of freedom by using a Feynman path integral representation. As far as the domain wall motion is concerned some efforts have been also made for semirings [33]. It has been also proposed to take into account the inhomogeneous anisotropy, which arises naturally in magnetic nanoring [34, 35, 35]. It was shown that the effect of the resulting Berry phase of electrons is quite similar to that of an external field affecting the equilibrium magnetization profile.

\section{Wires}

2.1 Theoretical model We consider first a long magnetic wire with two DWs, down which a current, $I$, is passed (as shown schematically in Fig. 1). Assuming that the distance $z_{0}$ between DWs is larger than the phase coherence length $L_{\phi}$, we can consider them as two independent scatterers, but for $z_{0} \lesssim L_{\phi}$ the current transmission mediates DW coupling. For definiteness, we assume that one of the DWs (located at $z=0$ ) is pinned, e.g. by a geometric constriction, and concentrate on the effect of the current on the second DW, initially (i.e., for $I=0$ ) located at $z=z_{0}$. For $I=0$ each DW has an extension $L$. We model the one-dimensional wire with the following Hamiltonian, $\bar{H}$, of noninteracting electrons coupled (with a coupling constant $J$ ) to a spatially non-uniform magnetization (DW) profile $\mathbf{M}(z)$ :

$$
\bar{H}=\int d z a_{\alpha}^{\dagger}(z)\left[-\frac{\delta_{\alpha \beta} \partial_{z}^{2}}{2 m}-J \boldsymbol{\sigma}_{\alpha \beta} \cdot \mathbf{M}(z)\right] a_{\beta}(z),
$$

(we use units with $\hbar=1$ ). Here $a_{\alpha}^{\dagger}$ and $a_{\alpha}$ are the creation and annihilation operators of electrons with spin $\alpha$. We use a local unitary transformation $T(z)$ [36, 37, 38] (often referred to as a gauge transformation) which transforms the second term with nonuniform magnetization into the constant Zeeman splitting term and an additional spindependent spatially varying potential $U_{\alpha \beta}(z)$. For $k_{F} L \gtrsim$ 1 this potential can be treated perturbatively [36, 37, 38, 39] ( $k_{F}$ is the Fermi wave vector) whereas the sharp domain walls, i.e. when $k_{F} L<1$, require a different formalism [40].

The matrix $T(z)$ is defined by $T^{\dagger}(z) \boldsymbol{\sigma} \cdot \mathbf{n}(z) T(z)=$ $\sigma^{z}$, where $\mathbf{n}$ is the unit vector along $\mathbf{M}, \mathbf{M}(z)=M \mathbf{n}(z)$. Then the transformed Hamiltonian $H=T^{\dagger}(z) \bar{H} T(z)$ is

$$
H=\int d z a_{\alpha}^{\dagger}(z)\left[-\frac{\delta_{\alpha \beta} \partial_{z}^{2}}{2 m}+U_{\alpha \beta}(z)-J M \sigma_{\alpha \beta}^{z}\right] a_{\beta}(z) .
$$

For a wire with two DWs we can parameterize the magnetization profile by two angles $\varphi(z)$ and $\theta(z)$ (cf. Fig. 11)

$$
\begin{aligned}
& \mathbf{n}(z)=(\cos \theta \sin \varphi, \sin \theta \sin \varphi, \cos \varphi), \\
& \varphi(z)=\underbrace{\cos ^{-1}\left(\tanh \left[\frac{z}{L}\right]\right)}_{=-\varphi_{1}(z)}+\underbrace{\cos ^{-1}\left(\tanh \left[\frac{z-z_{0}}{L}\right]\right)}_{=-\varphi_{2}(z)}
\end{aligned}
$$

(for details see Ref. [10]). The relative orientation between the two DWs (situated, respectively, at $z=0$ and $z=z_{0}$ ) is set by angle $\theta(z)$. We set $\theta_{1}=0$ at the first DW and $\theta_{2}=\theta_{0}$ around the second one (see Fig. 1). In the following we consider the case of $z_{0}>L$, which can be 
treated perturbatively as the coupling of the DWs is relatively small. Then we can write $U(z) \approx U_{1}(z)+U_{2}(z)$, where $(j=1,2)$

$$
\begin{aligned}
U_{j}(z)= & \frac{\left[\varphi_{j}^{\prime}(z)\right]^{2}}{8 m}+i \sigma^{y}\left[\frac{\varphi_{j}^{\prime \prime}(z)}{4 m}+\frac{\varphi_{j}^{\prime}(z) \partial_{z}}{2 m}\right] \cos \theta_{j}(z) \\
& -i \sigma^{x}\left[\frac{\varphi_{j}^{\prime \prime}(z)}{4 m}+\frac{\varphi_{j}^{\prime}(z) \partial_{z}}{2 m}\right] \sin \theta_{j}(z)
\end{aligned}
$$

Note that this approach is generalizable to any number of DWs which are sufficiently far apart.

As shown in Refs. [37, 38, 39], for a single DW and $k_{F} L \geq 1$ a perturbative approach is appropriate for treating the electron scattering from the DW potential (5). Thus, assuming $\psi^{0}(z)$ to be the wave function of electron with energy $\varepsilon$ in the wire without potential (5), we find the firstorder correction due to the perturbation $U_{1}(z)$, i.e. due to scattering from the first DW, as

$\delta \psi_{\varepsilon}(z)=\int_{-\infty}^{\infty} d z^{\prime} G_{\varepsilon}\left(z, z^{\prime}\right) U_{1}\left(z^{\prime}\right) \psi^{0}\left(z^{\prime}\right)$.

The Green's function $G_{\varepsilon}\left(z, z^{\prime}\right)$ corresponds to the unperturbed Hamiltonian (2) with $U(z)=0$. It is diagonal in the spin space with elements

$G_{\varepsilon \sigma}\left(z, z^{\prime}\right)=-\frac{i m}{k_{\sigma}} e^{i k_{\sigma}\left|z-z^{\prime}\right|}$,

where $k_{\sigma} \approx k_{\sigma}^{0}+\frac{i}{2 \tau_{\sigma}} \frac{m}{k_{\sigma}^{0}}$ for lifetimes $\tau_{\sigma} \gg \varepsilon_{F}^{-1}$, and $k_{\uparrow, \downarrow}^{0}=[2 m(\varepsilon+\mu \pm J M)]^{1 / 2}$.

The interaction energy of the two DWs due to the single scattered state $\psi_{\varepsilon \sigma}(z)=\psi_{\varepsilon \sigma}^{0}(z)+\delta \psi_{\varepsilon \sigma}(z)$ is

$\Delta E_{\sigma}=\int_{-\infty}^{\infty} d z \delta \psi_{\varepsilon \sigma}^{\dagger}(z) U_{2}(z) \delta \psi_{\varepsilon \sigma}(z)$

To find the total interaction energy we should sum up the contributions of all scattering states in the energy range between $\varepsilon_{F}$ and $\varepsilon_{F}+e \Delta \phi / 2$, for an applied voltage $e \Delta \phi / 2 \ll \varepsilon_{F}$. Then we find the current-induced coupling of the DWs:

$\Delta E=\frac{e \Delta \phi}{\sqrt{2} \pi}\left(\frac{\Delta E_{\uparrow}}{v_{\uparrow}}+\frac{\Delta E_{\downarrow}}{v_{\downarrow}}\right)$,

where $v_{\sigma}=k_{\sigma}^{0} / m$ is the velocity of spin up and down electrons at the Fermi level.

For numerical calculations we use the parameters of magnetic semiconductors [7,41]. It should be noted that in the case of metallic nanowires the 1D limit is also achievable [42]. Here we take the parameters as in Ref. [7], i.e. $\lambda_{F}=6 \mathrm{~nm}$; an effective mass of $m=0.5 m_{e}\left(m_{e}\right.$ is free electron mass); $L=\lambda_{F} ; J M=15 \mathrm{meV} ; \varepsilon_{F}=83.7 \mathrm{meV}$; and $e \Delta \phi=0.1 \varepsilon_{F}$. We also assume relatively large mean free path of $l=500 \mathrm{~nm}$, corresponding to essential ordering of Mn ions. The width of the wall may be as small as the atomic size in the presence of constrictions [43, 44,5], hence well below the DW lengths in bulk materials. In such a situation, the interaction between the DWs increases due

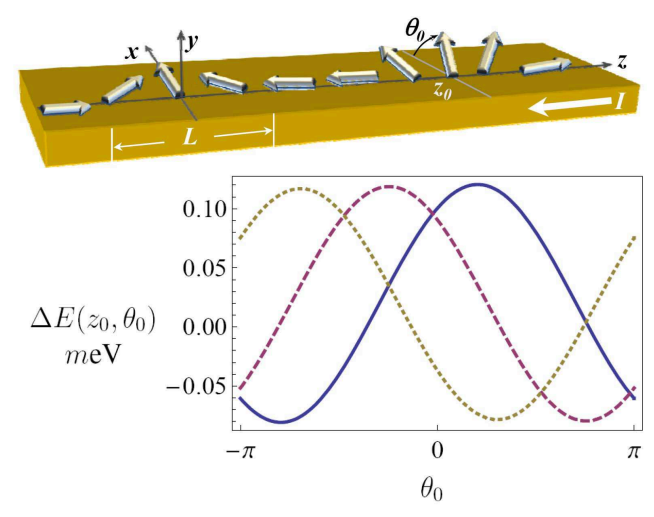

Figure 1 (Color online) Top panel: A schematic showing the DWs magnetization profile (thick arrows). $L$ is the DW width, $z_{0}$ and $\theta_{0}$ are respectively the DW position and orientation with respect to the DW at $z=0, I$ is the current direction. Lower panel: Interaction energy $\Delta E\left(z_{0}, \theta_{0}\right)$ as a function of $z_{0}$ and $\theta_{0}$. The solid curve is for $z_{0}=30 \mathrm{~nm}$, the dashed curve is for $z_{0}=37.5 \mathrm{~nm}$, and the dotted curve is for $z_{0}=45 \mathrm{~nm}$.

to strongly enhanced DW scattering [45, 46, 47,48]. The interaction energy shown in Fig. 1 depends periodically on the DWs relative angle $\theta_{0}$ and distance $z_{0}$. It results in an oscillating motion of the DW along the axis $z$ as well as an oscillating direction of DW polarization. In its turn this will have an effect on the spin torque driving the DW dynamics, which we will now focus on.

We find the spin density due to a single transmitted electron wave of spin $\sigma$ is

$\mathbf{S}_{\sigma}(z)=\psi_{\varepsilon \sigma}^{\dagger}(z) T(z) \boldsymbol{\sigma} T^{\dagger}(z) \psi_{\varepsilon \sigma}(z)$,

and the total current-induced spin density is [49]

$\mathbf{S}(z)=\frac{e \phi}{2 \pi}\left(\frac{\mathbf{S}_{\uparrow}}{v_{\uparrow}}+\frac{\mathbf{S}_{\downarrow}}{v_{\downarrow}}\right)$.

We find that the correction to the spin density follows the magnetization profile with additional Friedel oscillations, which are a superposition of two waves with periods $k_{F \uparrow}^{-1}$ and $k_{F \downarrow}^{-1}$. The oscillations in the spin density are smaller in magnitude than the overall spin density profile and decay with increasing $z$.

We calculate the current-induced torque acting on the magnetization at $z$, for the second DW located at $z_{0}$, from

$\Delta \mathbf{T}\left(z, z_{0}, \theta_{0}\right)=-\frac{\gamma J}{\sigma_{c s}} \mathbf{M}\left(z, z_{0}, \theta_{0}\right) \times \Delta \mathbf{S}\left(z, z_{0}, \theta_{0}\right)$,

where $\gamma=g \mu_{B}, g$ is the Landé factor and $\mu_{B}$ is the Bohr magneton. We assume a thin nanowire with a cross section of $\sigma_{c s}=100 \times 20 \mathrm{~nm}^{2}$ as in Ref. [7]. In Eq. (15) $\Delta \mathbf{S}$ is the correction to the electron spin density due to scattering. The calculated torque on the second DW is shown 


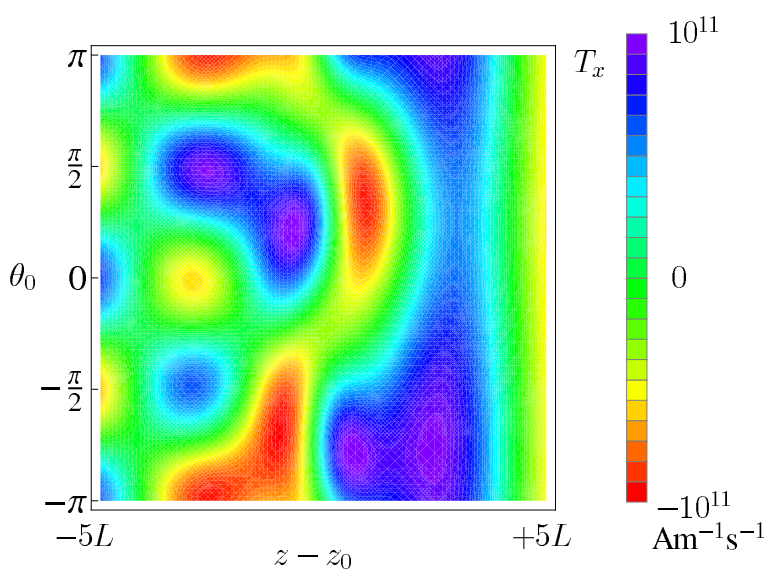

Figure 2 (Color online) The $x$-component of the currentinduced spin torque, as defined in Eq.12, acting at the second domain wall as a function of $z$ and $\theta_{0}$.

in Fig. 2 for the $x$-component, where $z_{0}=50 \mathrm{~L}$ and $M \approx 5.56 \times 10^{4} \mathrm{Am}^{-1}$ were used [41]. The correction to the spin torque shows that the force upon the DW depends strongly on their relative orientations.

To inspect the current-induced dynamics of the DW at $z=z_{0}$, we evaluate the accumulated spin density that acts on the DW at $z=z_{0}$. The DW magnetization dynamics are then modeled using the Landau-Lifshitz equation with the effect of magnetic anisotropy and damping:

$$
\begin{aligned}
\partial_{t} \mathbf{M}=-\frac{\gamma J}{\sigma_{c s}} \mathbf{M} \times \mathbf{S}[\mathbf{M}]+ & \frac{\gamma K^{\prime}}{M^{2}} \mathbf{M} \times \hat{\mathbf{x}} M_{x} \\
& +\frac{\alpha}{M} \mathbf{M} \times \partial_{t} \mathbf{M} .
\end{aligned}
$$

As an initial condition we assume that the magnetization profile in the wire without electric current is described by Eq. (4). We should note that the relative orientation of the walls at the start of motion does play a role in the type of motion we see. Here we present it for an arbitrary configuration.

We take the anisotropy constant to be $K^{\prime}=-10$, and therefore the $x$-axis to be a hard magnetization axis. Figure 3 shows the effects of anisotropy on the domain wall motion. The anisotropy dampens motion in the x-direction, thus exacerbating the $\mathrm{y}$ and $\mathrm{z}$ oscillations. This is also in contrast to the case where we ignore the first domain wall. In this case, although the anisotropy does introduce motion around the centre of the domain wall it does not involve a decaying $x$-component, see Fig. 4 .

Finally let us include weak Gilbert damping also ( $\alpha=$ 0.01 ), we then find a switch over between behaviour dominated by the anisotropy and behaviour dominated by the damping. See figure 5

\section{Quantum rings}

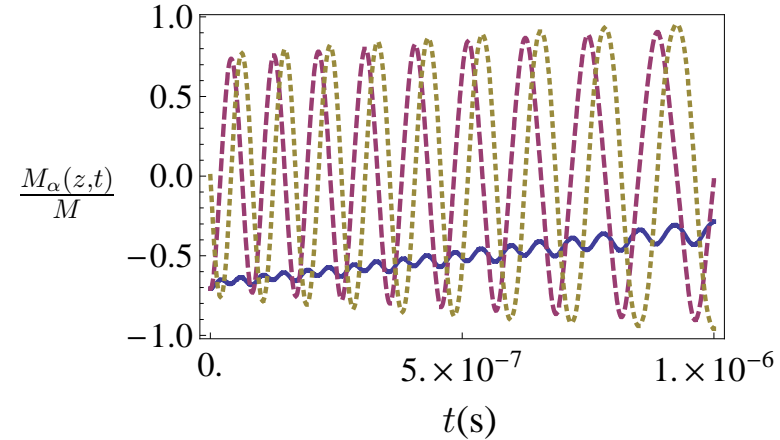

Figure 3 (Color online) The magnetization dynamics of the second DW when the anisotropy is included, see Eq. 13. The solid curve is the $x$-component, dashed the $y$-component, and dotted the $z$-component. Taken at the centre of the domain wall.

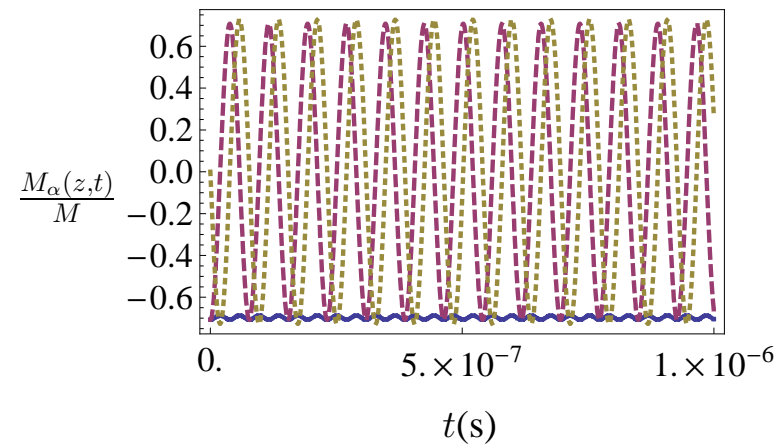

Figure 4 (Color online) The magnetization dynamics of the second DW without the influence of the first DW, when the anisotropy is included, see Eq. 13. The solid curve is the $x$-component, dashed the $y$-component, and dotted the $z$-component. Taken at the centre of the domain wall.

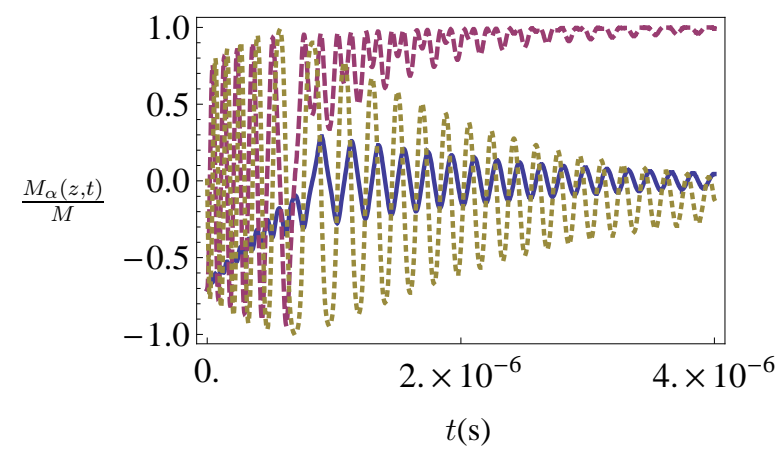

Figure 5 (Color online) The magnetization dynamics of the second DW when both anisotropy and damping are included, see Eq. 13 The solid curve is the $x$-component, dashed the $y$-component, and dotted the $z$-component. Taken at the centre of the domain wall. 
3.1 Model The following Hamiltonian

$$
\begin{array}{r}
\hat{H}=-\frac{1}{2 m}\left[\frac{1}{r} \partial_{r}\left(r \partial_{r}\right)+\frac{1}{r^{2}}\left(\partial_{\theta}-i \frac{\phi}{\phi_{0}}\right)^{2}\right] \\
-J M \hat{\mathbf{e}}_{\theta} \cdot \boldsymbol{\sigma}+V(r),
\end{array}
$$

describes a two dimensional ferromagnetic ring of electrons confined by some radial potential $V(r)$. Here we use the usual notations: $J$ is the exchange coupling constant and $m$ is the electron mass. In correspondance to the ring geometry it is convenient to use the polar coordinates. We denote

$\phi=\oint_{\gamma} \mathbf{A} \cdot d \mathbf{r}=\iint_{S} \mathbf{B} \cdot d \mathbf{S}$

$\phi_{0}=h / e$ is the magnetic flux quantum, $\mathbf{B}$ is the magnetic field and $\mathbf{A}$ is the vector potential. One can simplify Hamiltonian (14) making a local rotation in spin space to remove the $\theta$ dependence from the $J M$ term. We use the following unitary transformation:

$\mathbf{U}(\theta)=\frac{1}{\sqrt{2}}\left(\begin{array}{cc}i & 1 \\ -e^{i \theta} & -i e^{i \theta}\end{array}\right)$.

Note that this transformation preserves the same boundary conditions for the wavefunctions. The transformed Hamiltonian is $\hat{H}^{\prime}=\mathbf{U}^{\dagger}(\theta) \hat{H} \mathbf{U}(\theta)$ and the corresponding wave function is $\tilde{\boldsymbol{\psi}}(r, \theta)=\mathbf{U}^{\dagger}(\theta) \boldsymbol{\psi}(r, \theta)$. We obtain

$$
\begin{gathered}
\hat{H}^{\prime}=-\frac{1}{2 m}\left[\frac{1}{r} \partial_{r}\left(r \partial_{r}\right)+\frac{1}{r^{2}}\left(\partial_{\theta}-i \frac{\phi^{\prime}}{\phi_{0}}\right)^{2}\right. \\
\left.-\frac{1}{4 r^{2}}-\frac{i}{r^{2}} \boldsymbol{\sigma}_{y}\left(\partial_{\theta}-i \frac{\phi^{\prime}}{\phi_{0}}\right)\right]-J M \boldsymbol{\sigma}_{z}+V(r),
\end{gathered}
$$

where we introduced $\phi^{\prime}=\phi-\phi_{0} / 2$. The latter means the appearance of an additional phase of an electron $\phi_{0} / 2$ related to the ring geometry.

The angular dependence can be dealt with by a Fourier transform. We use the following definition:

$$
\begin{aligned}
\psi(r, \theta) & =\sum_{l=-\infty}^{\infty} e^{i l \theta} \boldsymbol{\psi}_{l}(r) \text { and } \\
\boldsymbol{\psi}_{l}(r) & =\int_{0}^{2 \pi} \frac{d \theta}{2 \pi} e^{-i l \theta} \boldsymbol{\psi}(r, \theta) .
\end{aligned}
$$

Finally we obtain the following transformed Hamiltonian:

$$
\begin{array}{r}
\hat{H}^{\prime}=-\frac{1}{2 m}\left[\frac{1}{r} \partial_{r}\left(r \partial_{r}\right)-\frac{1}{r^{2}} l_{\phi}^{\prime 2}-\frac{1}{4 r^{2}}+\frac{l_{\phi}^{\prime}}{r^{2}} \boldsymbol{\sigma}_{y}\right] \\
-J M \boldsymbol{\sigma}_{z}+V(r),
\end{array}
$$

where $l_{\phi}^{\prime}=l-\phi^{\prime} / \phi_{0}$. ¿From this point one can find the appropriate one-dimensional Hamiltonian [50] which can be further used to calculate the spin current in the ring without the DWs.

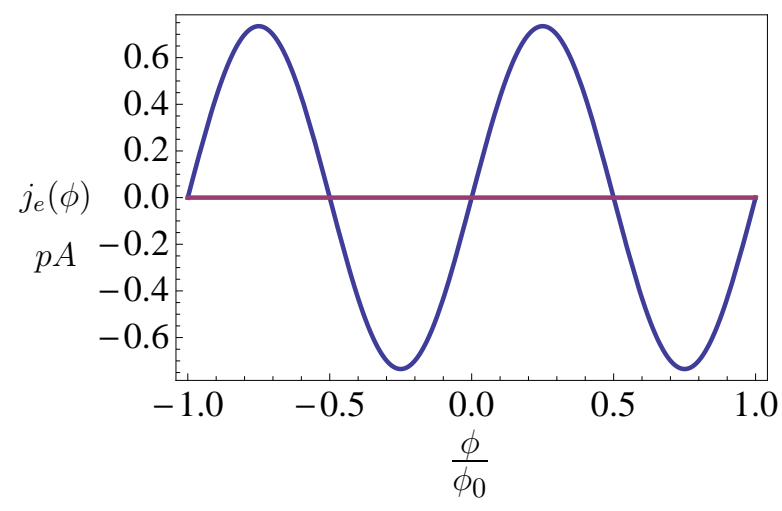

Figure 6 The persistent current, $j_{e}(\phi)$, in a onedimensional simple ferromagnetic ring.

3.2 Onions and vortices Once we understand the problem of the vortex state without the DWs, we can consider peeling back the layers of the onion state. The onion state refers to a ring in which there are two domain walls present, and hence two regions of oppositely directed magnetization. One can consider such state in the limits of sharp and adiabatic DWs (as compared to the electrons' wavelength). The following refers to the 1D model. Note that one should be careful in taking the 1D limit when a spin-orbit interaction is present [50].

3.3 Magnetic ring without the domain walls In this case we can make the transformation of Eq. (16). Then using $\hat{H}^{\prime}=\mathbf{U}^{\dagger}(\theta) \hat{H} \mathbf{U}(\theta)$ and $\tilde{\boldsymbol{\psi}}(\theta)=\mathbf{U}^{\dagger}(\theta) \boldsymbol{\psi}(\theta)$ we obtain

$$
\begin{aligned}
\hat{H}^{\prime} & =-\frac{1}{2 m \rho^{2}}\left[\partial_{\theta}-i \frac{\phi^{\prime}}{\phi_{0}}\right]^{2}+\frac{1}{4} \frac{1}{2 m \rho^{2}} \\
& +i \boldsymbol{\sigma}_{y} \frac{1}{2 m \rho^{2}}\left[\partial_{\theta}-i \frac{\phi^{\prime}}{\phi_{0}}\right]-J M \boldsymbol{\sigma}_{z} .
\end{aligned}
$$

After the Fourier transform we diagonalize the Hamiltonian; $\hat{H}^{\prime \prime}=\mathbf{N}^{\dagger}(l) \hat{H}^{\prime} \mathbf{N}(l)$ and $\tilde{\tilde{\boldsymbol{\psi}}}(l)=\mathbf{N}^{\dagger}(l) \tilde{\boldsymbol{\psi}}(l)$.

$\mathbf{N}(l)=\frac{1}{\sqrt{1-A_{l^{\prime}}^{+2}}}\left(\begin{array}{cc}1 & i A_{l^{\prime}}^{+} \\ A_{l^{\prime}}^{+} & i\end{array}\right)$.

$A_{l^{\prime}}^{+}=-i l_{\phi}^{\prime} /\left(\sqrt{l_{\phi}^{\prime 2}+\mu^{2}}-\mu\right)$ with $\mu=2 J M m \rho^{2}$. Then we obtain the exact formulas for the energy spectrum

$$
\begin{aligned}
& \varepsilon_{l(1,2)}=\frac{1}{2 m \rho^{2}}\left[l_{\phi}^{\prime 2}+\frac{1}{4}\right. \\
& \left.\mp\left(\mu \frac{1+A_{l^{\prime}}^{+2}}{1-A_{l^{\prime}}^{+^{2}}}-2 i l_{\phi}^{\prime} \frac{A_{l^{\prime}}^{+}}{1-A_{l^{\prime}}^{+^{2}}}\right)\right] .
\end{aligned}
$$

Now we can directly calculate the persistent current. Using

$j_{i}(\phi)=-\sum_{l} \frac{\partial \varepsilon_{l i}}{\partial \phi} f\left(\varepsilon_{l i}-\mu\right)$ 


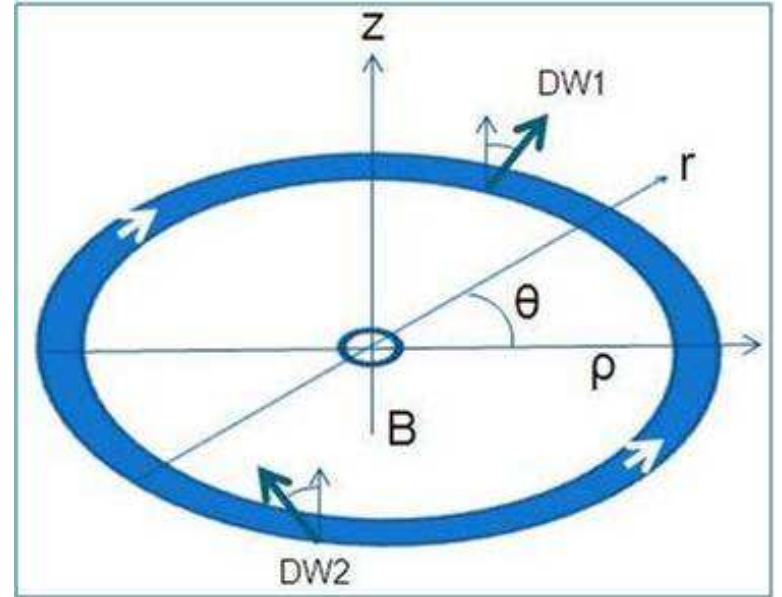

Figure 7 A schematic view of a ferromagnetic ring with an applied magnetic field.

(where $i=1,2$ ) we define the charge current as $j_{e}(\phi)=$ $j_{1}(\phi)+j_{2}(\phi)$. It is presented in Fig. 6 as a function of $\phi$.

\subsection{The onion state with adiabatic domain walls} In this section we use a modified version of our model for a wire with two domain walls [10] shown in Sec. 2. By mapping the circle onto a line, and directing the magnetization along $\hat{\mathbf{e}}_{\theta}$ instead of $\hat{\mathbf{e}}_{z}$, we can easily construct a suitable model. The mapping is given by

$x(\theta)=\frac{\rho}{L} \tan \left(\frac{\theta-\pi}{2}\right)$.

This mapping distorts the relative sizes of the domain walls depending on where on the ring they reside, and its accuracy increases with increasing the ring diameter.

$$
\begin{gathered}
\varphi(\theta)=\underbrace{\pi-\cos ^{-1}\left[\tanh \left[x(\theta)-x\left(\theta_{1}\right)\right]\right]}_{=\varphi_{1}(\theta)}+ \\
\underbrace{\pi-\cos ^{-1}\left[\tanh \left[x(\theta)-x\left(\theta_{2}\right)\right]\right]}_{=\varphi_{2}(\theta)} .
\end{gathered}
$$

where $\theta_{i}$ is the position in the ring of the $i$ th wall, which we take to be at an angle $\eta_{i}$ to the $z$-axis, see Fig. 7. The magnetization is then

$$
\begin{aligned}
\frac{\mathbf{M}(\theta)}{M}=\cos [\varphi(\theta)] \hat{\mathbf{e}}_{\theta} & +\sin [\eta(\theta)] \sin [\varphi(\theta)] \hat{\mathbf{e}}_{r} \\
& +\cos [\eta(\theta)] \sin [\varphi(\theta)] \hat{\mathbf{e}}_{z},
\end{aligned}
$$

and the Hamiltonian is given by

$$
\hat{H}=-\frac{1}{2 m \rho^{2}}\left[\partial_{\theta}-i \frac{\phi}{\phi_{0}}\right]^{2}-J \mathbf{M}(\theta) . \boldsymbol{\sigma} .
$$

where $\eta(\theta)$ is a function, which takes the value $\eta_{i}$ around the $i$ th wall and slowly interpolates between the walls.
We can locally rotate the spin direction as before. The resultant Hamiltonian,

$$
\begin{array}{r}
\hat{H}^{\prime}=-\frac{1}{2 m \rho^{2}}\left[\left(\partial_{\theta}-i \frac{\phi}{\phi_{0}}\right)^{2}-\frac{1}{4}-i\left(\partial_{\theta}-i \frac{\phi}{\phi_{0}}\right) \boldsymbol{\sigma}_{y}\right] \\
-J \tilde{\mathbf{M}}(\theta) \cdot \boldsymbol{\sigma}
\end{array}
$$

includes the following magnetization profile:

$$
\begin{aligned}
\frac{\tilde{\mathbf{M}}(\theta)}{M}=\cos [\varphi(\theta)] \hat{\mathbf{e}}_{z} & -\sin [\eta(\theta)] \sin [\varphi(\theta)] \hat{\mathbf{e}}_{x} \\
& +\cos [\eta(\theta)] \sin [\varphi(\theta)] \hat{\mathbf{e}}_{y} .
\end{aligned}
$$

After that we can make a gauge transformation to reduce the magnetization profile to a constant Zeeman term and an additional potential. However, the non-diagonal portions of the transformed Hamiltonian complicate significantly the exact calculation of the energy spectrum.

Therefore, we consider a simplified approach which is justified for large magnetic ring, $k \rho \gg 1$, where $k$ is the electron wave vector along the ring. Using Eq. (29) we write

$$
\begin{array}{r}
\hat{H}^{\prime}=-\frac{1}{2 m}\left[\left(\partial_{z}-\frac{i \phi}{\rho \phi_{0}}\right)^{2}-\frac{1}{4 \rho^{2}}-\frac{i}{\rho}\left(\partial_{z}-\frac{i \phi}{\rho \phi_{0}}\right) \boldsymbol{\sigma}_{y}\right] \\
-J \tilde{\mathbf{M}}(\theta) \cdot \boldsymbol{\sigma}
\end{array}
$$

where $z=\rho \theta$ is the coordinate along the wire. Due to the periodicity in angle $\theta$, the condition of periodicity $z \rightarrow$ $z+2 \pi \rho$ should be preserved. If $k \rho \gg 1$ the second term in Eq. (30) is small and can be taken into account perturbatively. If we neglect the small correction we come to the 1D Hamiltonian, which can be treated using the same methods as the linear model of Sec. 2. The main difference is that the gauge potential related to the DWs is an additional correction to the gauge related to the ring curvature and to the magnetic flux through the ring.

It should be noted that the ring topology allows only an even number of DWs. In the case of two DWs on the ring, the current-induced interaction between them can be described by the spin torque formula Eq. (12). On the other hand, the persistent current in the magnetic ring, Eq. 23), would necessarily induce the current-induced force and an interaction between the walls, which puts them both into a kind of circular motion. However, one can also expect that the damping associated with the DW motion would lead to a slow decay of the persistent current. We can assume that a slight pumping of power from an external source can make the motion of DWs on the ring non-decaying, which makes this problem attractive for possible applications.

4 Summary We have shown how the presence of more than one domain wall in a wire affects the domain wall dynamics and the energy of the electron system. We calculated the energy due to the current mediated coupling between the domain walls and demonstrated how the domain wall dynamics is modified by the current passing through a pair of the domain walls in the wire. 
Then we consider ferromagnetic nanorings with two domain walls. In this case the DW dynamics is also affected by the coupling between the walls. Besides this, there appear additional contributions to the gauge potential related to the ring geometry of the ferromagnetic state and to the magnetic flux through the ring. In the case of a relatively large ring with two domain walls, the problem can be solved as for the wire with the periodic condition for eigenfunctions, resulting in the quantization of values of the wavevector $k$ along the ring.

Acknowledgements This work is supported by DFG under Grant No. SPP 1165 and partly by the FCT Grant PTDC/FIS/70843/2006 in Portugal and by Polish Ministry of Science and Higher Education as a research project in years 2007 -2010 .

\section{References}

[1] M. Yamanouchi, D. Chiba, F. Matsukura, and H. Ohno, Nature 428, 539 (2004).

[2] A. Yamaguchi, T. Ono, S. Nasu, K. Miyake, K. Mibu, and T. Shinjo, Phys. Rev. Lett. 92, 077205 (2004).

[3] S. Parkin, M. Hayashi, and L. Thomas, Science 320, 190 (2008).

[4] M. Klaeui, J. Phys. Condens. Matter 20, 313001 (2008).

[5] U. Ebels, A. Radulescu, Y. Henry, L. Piraux, , and K. Ounadjela, Phys. Rev. Lett. 84, 983 (2000).

[6] H. Chopra and S. Hua, Phys. Rev. B 66, 020403 (2002).

[7] C. Rüster, T. Borzenko, C. Gould, G. Schmidt, L. Molenkamp, X. Liu, T. Wojtowicz, J. Furdyna, Z. Yu, and M. Flatté, Phys. Rev. Lett. 91, 216602 (2003).

[8] C. Vaz, T. Hayward, J. Llandro, F. Schackert, D. Morecroft, J. A. C. Bland, M. Kläui, M. Laufenberg, D. Backes, U. Rüdiger, F. J. Castano, C. A. Ross, L. J. Heyderman, F. Nolting, A. Locatelli, G. Faini, S. Cherifi, and W. Wernsdorfer, J. Phys. Condens. Matter 19, 255207 (2007).

[9] M. Kläui, J. Phys. Condens. Matter 20, 313001 (2008).

[10] N. Sedlmayr, V. K. Dugaev, and J. Berakdar, Phys. Rev. B 79, 174422 (2009).

[11] N. Sedlmayr and et al., J. Magn. Magn. Mater 322, 1419 (2010).

[12] N. Sedlmayr and J. Berakdar, Eur. Phys. Lett. 88, 57003 (2008).

[13] Y. Aharonov and D. Bohm, Phys. Rev. 115(3), 485-491 (1959).

[14] R. A. Webb, S. Washburn, C.P. Umbach, and R. B. Laibowitz, Phys. Rev. Lett. 54(25), 2696-2699 (1985).

[15] M. Büttiker, Y. Imry, and R. Landauer, Physics Letters A 96(7), 365-367 (1983).

[16] L. P. Lévy, G. Dolan, J. Dunsmuir, and H. Bouchiat, Phys. Rev. Lett. 64(17), 2074-2077 (1990).

[17] Y. Gefen, Y. Imry, and M. Y. Azbel, Phys. Rev. Lett. 52(2), 129-132 (1984).

[18] J. Slonczewski, J. Magn. Magn. Mater. L1, 159 (1996).

[19] L. Berger, Phys. Rev. B 54, 9353 (1996).

[20] J. Barnaś, A. Fert, M. Gmitra, I. Weymann, and V. Dugaev, Phys. Rev. B 72, 024426 (2005).

[21] J. Slonczewski, Phys. Rev. B 39, 6995 (1989).
[22] I. Theodonis, N. Kioussis, A. Kalitsov, M. Chshiev, and W. Butler, Phys. Rev. Lett. 97, 237205 (2006).

[23] S. Kasai, T. Niiyama, E. Saitoh, and H. Miyajima, Applied Physics Letters 81, 316 (2002).

[24] K. Sekiguchi, A. Yamaguchi, H. Miyajima, and A. Hirohata, Phys. Rev. B 77, 140401 (2008).

[25] M. Kläui, C. A. F. Vaz, J. Rothman, J. A.C. Bland, W. Wernsdorfer, G. Faini, and E. Cambril, Phys. Rev. Lett. 90(9), 097202 (2003).

[26] M. F. Lai, Z. H. Wei, C. R. Chang, J. C. Wu, J. H. Kuo, and J. Y. Lai, Phys. Rev. B 67(10), 104419 (2003).

[27] S. Jain, C. C. Wang, and A. O. Adeyeye, Nanotechnology 19, 085302 (2008).

[28] W. C. Tan and J. C. Inkson, Semicond. Sci. Technol. 11, 1635 (1996).

[29] N. Kim, G. Ihm, H. S. Sim, and K. J. Chang, Phys. Rev. B 60(12), 8767-8772 (1999).

[30] G. Tatara and B. Barbara, Phys. Rev. B 64(17), 172408 (2001).

[31] G. Tatara, H. Kohno, E. Bonet, and B. Barbara, Phys. Rev. B 69(5), 054420 (2004).

[32] D. Loss, P. Goldbart, and A. V. Balatsky, Phys. Rev. Lett. 65(13), 1655-1658 (1990).

[33] G. Meier, M. Bolte, . Merkt, B. Krüger, and D. Pfannkuche, J. Mag. Magnetic Materials 316, e966 (2007).

[34] P. Bruno and V. K. Dugaev, Phys. Rev. B 72, 241302(R) (2005).

[35] V. K. Dugaev and P. Bruno, Phys. Rev. B 75, 201301(R) (2007).

[36] V. Korenman, J. Murray, and R. Prange, PRB 16, 4032 (1977).

[37] G. Tatara and H. Fukuyama, PRL 78, 3773 (1997).

[38] V. Dugaev, J. Barnaś, A. Łusakowski, and L. Turski, Phys. Rev. B 65, 224419 (2002).

[39] V. Dugaev, V. Vieira, P. Sacramento, J. Barnaś, M. Araújo, and J. Berakdar, Int. J. Mod. Phys. 21, 1659 (2007).

[40] V. Dugaev, J. Berakdar, and J. Barnaś, Phys. Rev. B 86, 104434 (2003).

[41] A. Sugawara, H. Kasai, A. Tonomura, P. Brown, R. Campion, K. Edmonds, B. Gallagher, J. Zeman, and T. Jungwirth, Phys. Rev. Lett. 100, 0477202 (2008).

[42] J. Schäfer, C. Blumenstein, S. Meyer, M. Wisniewski, and R. Claessen, Phys. Rev. Lett. 101, 236802 (2008).

[43] P. Bruno, Phys. Rev. Lett. 83, 2425 (1999).

[44] O. Pietzsch, A. Kubetzka, M. Bode, and R. Wiesendanger, Phys. Rev. Lett. 84, 5212 (2000).

[45] M. Araújo, V. Dugaev, V. Vieira, J. Berakdar, and J. Barnaś, Phys. Rev. B 74, 224429 (2006).

[46] V. Dugaev, V. Vieira, P. Sacramento, J. Barnaś, M. Araújo, and J. Berakdar, Phys. Rev. B 74, 054403 (2006).

[47] V. Dugaev, J. Berakdar, and J. Barnaś, Phys. Rev. Lett. 96, 047208 (2006).

[48] V. Dugaev, J. Barnaś, and J. Berakdar, Phys. Rev. B 71, 024430 (2005).

[49] V. Dugaev, J. Barnaś, and J. Berakdar, J. Phys. A 36, 9263 (2003).

[50] F.E. Meijer, A.F. Morpurgo, and T. M. Klapwijk, Phys. Rev. B 66(3), 033107 (2002). 